\title{
Predictions from heavy new physics interpretation of the top forward-backward asymmetry
}

\author{
Cédric Delaunay, ${ }^{a}$ Oram Gedalia, ${ }^{b}$ Yonit Hochberg $^{b}$ and Yotam Soreq $^{b}$ \\ ${ }^{a}$ Theory Division, Physics Department, CERN, \\ CH-1211 Geneva 23, Switzerland \\ ${ }^{b}$ Department of Particle Physics and Astrophysics, Weizmann Institute of Science, \\ Rehovot 76100, Israel \\ E-mail: cedric.delaunay@cern.ch, oram.gedalia@weizmann.ac.il, \\ yonit.hochberg@weizmann.ac.il, yotam.soreq@weizmann.ac.il
}

ABSTRACT: We derive generic predictions at hadron colliders from the large forwardbackward asymmetry observed at the Tevatron, assuming the latter arises from heavy new physics beyond the Standard Model. We use an effective field theory approach to characterize the associated unknown dynamics. By fitting the Tevatron $t \bar{t}$ data we derive constraints on the form of the new physics. Furthermore, we show that heavy new physics explaining the Tevatron data generically enhances at high invariant masses both the top pair production cross section and the charge asymmetry at the LHC. This enhancement can be within the sensitivity of the $8 \mathrm{TeV}$ run, such that the $2012 \mathrm{LHC}$ data should be able to exclude a large class of models of heavy new physics or provide hints for its presence. The same new physics implies a contribution to the forward-backward asymmetry in bottom pair production at low invariant masses of order a permil at most.

Keywords: Phenomenological Models, Hadronic Colliders

ARXIV EPRINT: 1207.0740 


\section{Contents}

1 Introduction 1

2 Effective field theory approach $\quad 2$

3 Global fit to the top Tevatron data 4

4 LHC predictions and constraints 5

$\begin{array}{lll}5 & \text { Dijet constraints } & 7\end{array}$

$6 \quad$ Implications for $b \bar{b}$ production asymmetry 9

$\begin{array}{llr}7 & \text { Conclusions } & 10\end{array}$

\section{Introduction}

Physics beyond the Standard Model (SM) has thus far eluded experimental discovery. The $7 \mathrm{TeV}$ run of the LHC has not produced any signs of new TeV scale states. Nevertheless, New Physics (NP) around the $\mathrm{TeV}$ scale is required by naturalness of the electroweak scale, which in the SM is mainly destabilized by the heaviness of the top quark. It is thus sensible to expect that a (technically) natural UV completion of the SM extends the top sector, such that top quark observables at hadron colliders might deviate from their SM predictions. In this theoretical context, the observation of an anomalously large forwardbackward asymmetry in top pair production at the Tevatron is intriguing.

At the inclusive level, both CDF and D0 have been consistently reporting values exceeding the SM prediction by $\sim 2 \sigma$ in various channels [1-4]. Most striking is the evidence for a growth of the asymmetry with $M_{t \bar{t}}$ reported by CDF [4], which is well illustrated by their measurement of the asymmetry above $450 \mathrm{GeV}$ :

$$
A_{\mathrm{FB}}^{t \bar{t}}\left(M_{t \bar{t}} \geq 450 \mathrm{GeV}\right)=+0.296 \pm 0.059 \text { (stat.) } \pm 0.031 \text { (syst.) } .
$$

Although the central value is lower than their previous result [1], it is still $\sim 3 \sigma$ above the SM prediction of $\sim 0.1$ [4]. The latter might be underestimated due to higher-order QCD and electroweak effects [5] (see also the impact of the renormalization scale uncertainty [6]), yet the above series of observations is certainly worth exploring in the context of NP beyond the SM.

There are two LHC measurements of direct relevance to the top $A_{\mathrm{FB}}^{t \bar{t}}$ : the $t \bar{t}$ differential cross section as a function of $M_{t \bar{t}}$ and the charge asymmetry $\left(A_{\mathrm{C}}\right)$ in $t \bar{t}$ production. 
The $7 \mathrm{TeV}$ LHC run has already provided preliminary measurements of these two observables. For instance, the CMS collaboration recently measured the charge asymmetry at the inclusive level,

$$
\left(A_{\mathrm{C}}\right)^{\mathrm{inc}}=+0.004 \pm 0.010 \text { (stat.) } \pm 0.011 \text { (syst.) }
$$

as well as its distribution with $M_{t \bar{t}}$, based on $5.0 \mathrm{fb}^{-1}$ of data [7]. Similar results were obtained by the ATLAS collaboration $[8,9]$. These results are consistent with the SM expectations. Measurements of the differential cross section at the LHC have been carried out using conventional top-reconstruction techniques (see e.g. [10]), and good agreement with the QCD prediction below $M_{t \bar{t}} \lesssim 700 \mathrm{GeV}$ is found.

We interpret the discrepancy between the Tevatron $A_{\mathrm{FB}}^{t \bar{t}}$ data and the SM expectations as originating from heavy NP. (For previous relevant works see [11-16], and for a review of various NP models see e.g. [17].) We assume that the scale of the new physics is well above the scale $M_{t \bar{t}}$ that is relevant to the Tevatron measurements, and is such that the heavy NP is not produced on-shell at the LHC running at center-of-mass energies up to $8 \mathrm{TeV}$. These effects are well captured by an Effective Field Theory (EFT) expansion below the pole of the NP states, and can therefore be studied completely generically without specifying any UV completion. Moreover, the wait for signals of NP at the LHC together with electroweak precision data from LEP [18-20] imply that heavy NP at the few $\mathrm{TeV}$ scale is a motivated framework.

The main goal of the present paper is to demonstrate within the EFT framework that the heavy NP explanation of the anomalous $A_{\mathrm{FB}}^{t \bar{t}}$ is still largely consistent with the 2011 LHC measurements, and that the LHC run at $8 \mathrm{TeV}$ should have enough sensitivity to observe the effects of the heavy NP or to exclude most of the parameter space accommodating the Tevatron data.

The paper is organized as follows. Section 2 lists the relevant EFT operators. In section 3 we perform a global fit of the operators to the most updated Tevatron $t \bar{t}$ data set. Section 4 compares the result of the global fit to present ATLAS and CMS data, and gives predictions for the differential $t \bar{t}$ spectrum and charge asymmetry at the LHC. In sections 5 and 6 we comment on the possible interplay of the top data with dijet production at the LHC and forward-backward $b \bar{b}$ production asymmetry at the Tevatron, respectively. We conclude in section 7 .

\section{Effective field theory approach}

In order to collectively describe heavy NP, we use a set of effective operators, under the assumptions that the scale $\Lambda$ characterizing NP is significantly higher than the top pair invariant mass in the relevant measurements. These operators must relate an initial $u \bar{u}$ state to a final $t \bar{t}$ state, and as such appear at dimension six and higher. Operators leading from $d \bar{d}$ to $t \bar{t}$ are suppressed at the Tevatron due to the small $d \bar{d}$ luminosity. Since our goal is to use the Tevatron data to make predictions for the LHC, these operators are henceforth omitted from the analysis. For use of these operators to ameliorate potential tension between Tevatron and LHC data see [21]. 
The relevant dimension six operators are made of four-fermion contact terms. The operators with vector and axial-vector structure are:

$$
\begin{aligned}
\mathcal{O}_{V}^{1,8} & =\left(\bar{u} \gamma_{\mu} T_{1,8} u\right)\left(\bar{t} \gamma^{\mu} T_{1,8} t\right), & \mathcal{O}_{A}^{1,8} & =\left(\bar{u} \gamma_{\mu} \gamma^{5} T_{1,8} u\right)\left(\bar{t} \gamma^{\mu} \gamma^{5} T_{1,8} t\right), \\
\mathcal{O}_{A V}^{1,8} & =\left(\bar{u} \gamma_{\mu} \gamma^{5} T_{1,8} u\right)\left(\bar{t} \gamma^{\mu} T_{1,8} t\right), & \mathcal{O}_{V A}^{1,8} & =\left(\bar{u} \gamma_{\mu} T_{1,8} u\right)\left(\bar{t} \gamma^{\mu} \gamma^{5} T_{1,8} t\right) .
\end{aligned}
$$

The scalar and tensor operators are:

$$
\begin{array}{rlrl}
\mathcal{O}_{S}^{1,8} & =\left(\bar{u} T_{1,8} u\right)\left(\bar{t} T_{1,8} t\right), & & \mathcal{O}_{P}^{1,8}=\left(\bar{u} T_{1,8} \gamma^{5} u\right)\left(\bar{t} T_{1,8} \gamma^{5} t\right), \\
\mathcal{O}_{S P}^{1,8} & =i\left(\bar{u} T_{1,8} u\right)\left(\bar{t} T_{1,8} \gamma^{5} t\right), & & \mathcal{O}_{P S}^{1,8}=i\left(\bar{u} T_{1,8} \gamma^{5} u\right)\left(\bar{t} T_{1,8} t\right), \\
\mathcal{O}_{T}^{1,8} & =\left(\bar{u} T_{1,8} \sigma^{\mu \nu} u\right)\left(\bar{t} T_{1,8} \sigma_{\mu \nu} t\right), &
\end{array}
$$

with $T_{1} \equiv 1$ and $T_{8} \equiv T^{a}$. The operators $\mathcal{O}_{V, A}^{8}$ in eq. (2.1) interfere with the SM and thus contribute to $u \bar{u} \rightarrow t \bar{t}$ processes at $\mathcal{O}\left(\alpha_{s} / \Lambda^{2}\right)$, while the rest of the operators in eqs. (2.1) and $(2.2)$ only contribute at $\mathcal{O}\left(1 / \Lambda^{4}\right)$.

In principle, there are also chromomagnetic dipole operators involving the gluon field strength which contribute to $u \bar{u} \rightarrow t \bar{t}$. Their contribution at $\mathcal{O}\left(1 / \Lambda^{4}\right)$ is suppressed by at least $\left(m_{t} / \Lambda\right)$ compared to their dominant $1 / \Lambda^{2}$ effects [15]. Their interference level contribution has the same shape as the QCD $M_{t \bar{t}}$ distribution [12], and so these operators are strongly constrained by $t \bar{t}$ cross section measurements at the Tevatron. We thus neglect the chromomagnetic operators from further discussion. Dimension eight operators interfering with the SM are subdominant compared to the effects that we consider when the NP couplings are sizable at the scale where NP is on-shell [15], as is typically required by the large $A_{\mathrm{FB}}^{t \bar{t}}$ measurement. As such, they are henceforth omitted.

The total effective Lagrangian which we consider is then given by

$$
\mathcal{L}_{\text {eff }}=\sum_{i} \frac{C_{i}}{\Lambda^{2}} \mathcal{O}_{i} \equiv \sum_{i} c_{i} \mathcal{O}_{i}
$$

where the sum is over the operators in eq. (2.1) and (2.2) and $c_{i}$ are of dimension [mass] $]^{-2}$. This effective description has a limited range of validity in terms of energies. The range is limited from above by the breakdown of the perturbative EFT description. Naive dimensional analysis dictates $C_{i} \lesssim 16 \pi^{2}$, and plugging in the typical value for $c_{A}^{8}$ suggested by the result of our fit (see section 3) leads to

$$
\Lambda<\mathcal{O}(10 \mathrm{TeV})
$$

The lower edge of the validity range is around $1 \mathrm{TeV}$, above the typical scale of the relevant observables.

In order to minimize the impact of next to leading order corrections to these NP contributions, we normalize the latter to the SM one in all calculations. We assume that the $K$-factors are universal, so that the NP/SM ratios at leading order and next to leading order are the same. For an extended discussion, see [14, 15]. 


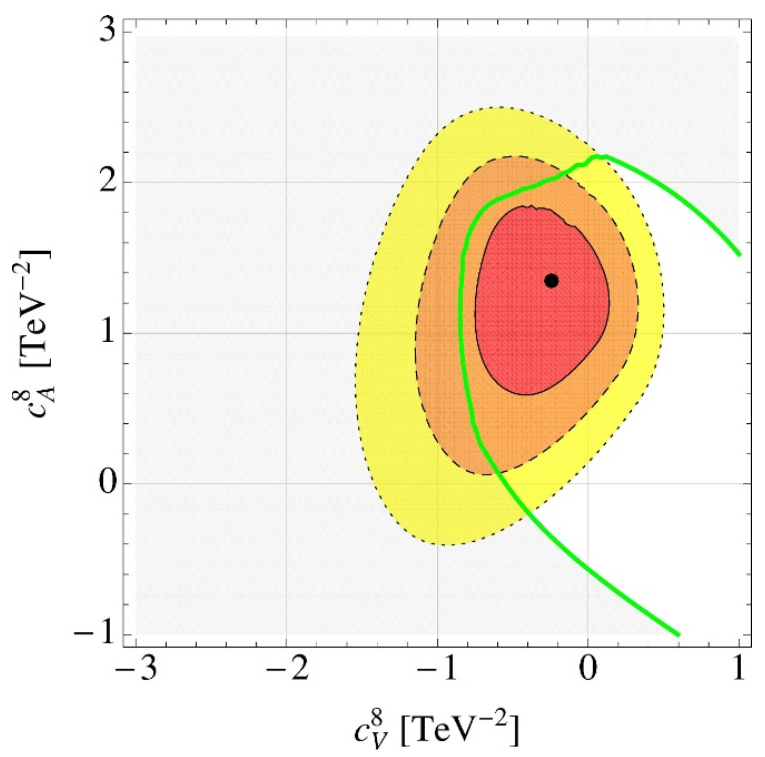

Figure 1. The result of the $\chi^{2}$ fit to the top related Tevatron data, presented in the plane of $c_{V}^{8}$ and $c_{A}^{8}$, while marginalizing over the rest of the operators. The red, orange and yellow shaded regions correspond to $1 \sigma, 2 \sigma$ and $3 \sigma$, respectively, and the black dot stands for the best fit point. The light gray shaded region to the left of the green contour is excluded by the experimental bound on the $t \bar{t}$ cross section for $M_{t \bar{t}}>1 \mathrm{TeV}$, eq. (4.1).

\section{Global fit to the top Tevatron data}

The NP contributions described by the operators defined above can be used to fit the Tevatron data on top related observables. We begin by describing the relevant measurements. We avoid the need to deal with correlations in the data by considering only measurements which can be treated as uncorrelated. We thus use the following Tevatron data and the corresponding SM estimates:

- inclusive $t \bar{t}$ cross section from D0 [22] and the QCD NNLO calculation [23].

- Differential $t \bar{t}$ cross section as a function of $M_{t \bar{t}}$ from CDF [24] (table 3, all bins but the first), to be compared with the approximate NNLO calculation of [25] (figure 12).

- Inclusive $t \bar{t}$ forward-backward asymmetry as measured by D0 in the lepton+jet channel [3] and by CDF in the dilepton channel [2], and the corresponding NLO QCD+EW estimate quoted by CDF in [4].

- Differential $t \bar{t}$ forward-backward asymmetry as a function of $M_{t \bar{t}}$ from CDF and the NLO QCD+EW estimate [4] (table XVII).

We fit the Wilson coefficients of the Lagrangian eq. (2.3) to the Tevatron data listed above using a $\chi^{2}$ analysis, where the observables are calculated using the MSTW parton distribution functions [26] at leading order. Since large interference effects are required by the data [27] we derive confidence level contours in the plane of the two interfering operators $\mathcal{O}_{V, A}^{8}$ by marginalizing over all the non-interfering operators. Note that in practice the 
full $t \bar{t}$ differential cross section contains only 6 independent terms, and so the list of Wilson coefficients can be combined into 6 parameters. The result is presented in figure 1 . The best fit point corresponds to $c_{A}^{8}=1.35 \mathrm{TeV}^{-2}, c_{V}^{8}=-0.24 \mathrm{TeV}^{-2}$ and mild contributions from the non-interfering operators. The goodness-of-fit is 0.88 .

We learn that heavy NP which does not interfere with the $\mathrm{SM}\left(c_{V}^{8}=c_{A}^{8}=0\right)$ is in $\sim 3 \sigma$ tension with the data. Furthermore, the absence of the axial-octet operator $\mathcal{O}_{A}^{8}$ is in $\sim 2 \sigma$ tension with the data. Qualitatively, the non-interfering operators do not relax the need for interference mainly due to their rapid growth with $M_{t \bar{t}}$, which makes them strongly constrained by the measurement of the differential cross-section in the hard regime.

If the heavy NP generates a single operator of definite chirality for each color structure, then a positive $A_{\mathrm{FB}}^{t \bar{t}}$ can be induced through either left-handed up quarks and right-handed top quarks or vice versa. In the vector-axial basis defined in eq. (2.1) this corresponds in particular to $c_{V}^{8}=-c_{A}^{8}$, affecting at the interference level both the $t \bar{t}$ cross section and the asymmetry. Indeed we find that a similar $\chi^{2}$ fit in the chiral case is worse by at least $2 \sigma$ than the best fit point in the general case. For illustration, at the minimum of $\chi^{2}$ in the chiral case, we find $A_{\mathrm{FB}}^{t \bar{t}} \lesssim 20 \%$ and $\sim 2 \sigma$ tension with the cross section.

In principle, this analysis does not rely on any specific UV completion. Yet, specific models of heavy NP can be mapped to the EFT description of section 2. To this end, we use the list of models presented in ref. [28], considering the addition of each new particle individually. (These models were examined in the context of the forward-backward asymmetry in e.g. [16].) Since most of the models contribute to the set of EFT operators via a single chirality structure, we find that they are in tension with the data, as mentioned above. The only model of a single new particle that can give independent values to $c_{A}^{8}$ and $c_{V}^{8}$, therefore providing a good match to the fit, is of a heavy color octet and $\mathrm{SU}(2)_{L}$ singlet, namely an axigluon.

\section{LHC predictions and constraints}

We now discuss predictions for LHC measurements arising from the EFT description of the top Tevatron data. We first compare the above fit results with existing LHC data based on the $7 \mathrm{TeV}$ run, and then provide predictions for the 2012 run at $8 \mathrm{TeV}$.

Regarding the $t \bar{t}$ cross section at the LHC, the heavy NP explanation of the Tevatron data can significantly affect the top pair spectrum only at invariant masses above $\sim 1 \mathrm{TeV}[15]$. In this regime, top decay products are highly collimated such that they merge into a single jet, and their reconstruction therefore requires dedicated jet substructure techniques. Currently, there is no partonic level result for the shape of the $t \bar{t}$ spectrum in this boosted regime. However, CMS has recently published an upper bound on the enhancement in the $t \bar{t}$ cross section integrated over $M_{t \bar{t}}>1 \mathrm{TeV}$ based on the all hadronic channel [29]:

$$
\mathcal{S} \equiv \frac{\int_{M_{t \bar{t}}>1 \mathrm{TeV}} \frac{\mathrm{d} \sigma_{\mathrm{SM}+\mathrm{NP}}}{\mathrm{d} M_{t \bar{t}}} \mathrm{~d} M_{t \bar{t}}}{\int_{M_{t \bar{t}}>1 \mathrm{TeV}} \frac{\mathrm{d} \sigma_{\mathrm{SM}}}{\mathrm{d} M_{t \bar{t}}} \mathrm{~d} M_{t \bar{t}}}<2.6,
$$

at $95 \%$ confidence level. This bound can be used to constrain the EFT parameter space, as depicted in figure 1. It is evident that the bulk of the parameter space that accounts 

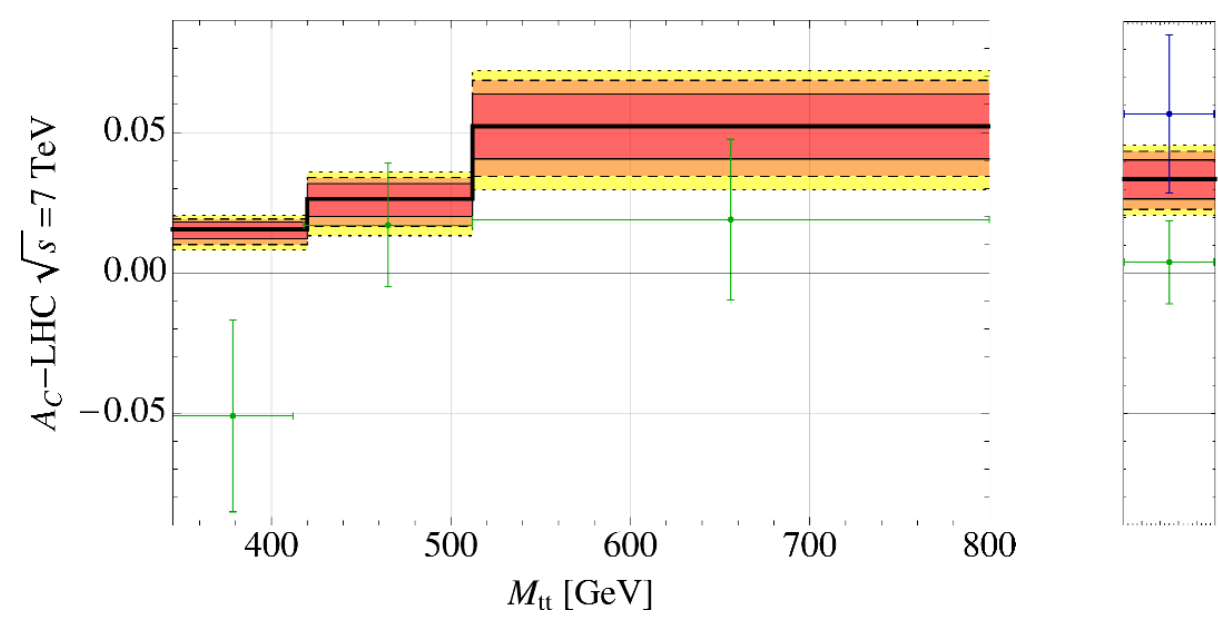

Figure 2. Comparison of the EFT fit to the differential (left) and inclusive (right) charge asymmetry to the LHC data. The red, orange and yellow shaded regions correspond to $1 \sigma, 2 \sigma$ and $3 \sigma$ of the global fit to Tevatron data, respectively, and the green points and error bars describe the CMS measurement [7]. The inclusive charge asymmetry measured by ATLAS [9] is shown in blue.

for the Tevatron data is left intact, with a prediction of $\mathcal{S} \simeq 1.8$ for the best fit point reported above. Future improvement of this measurement might further constrain the EFT parameters.

Next we consider the measured charge asymmetry in eq. (1.2), including the differential data as a function of $M_{t \bar{t}}$. Figure 2 presents the charge asymmetry stemming from the fit, compared to the CMS [7] and ATLAS [9] data. Currently, there is no strong tension between the Tevatron measurement of the top forward-backward asymmetry and the LHC charge asymmetry data within the EFT framework. It will be interesting to see how future updates of the data affect the EFT paradigm.

In accordance with the above results, we have verified that including the charge asymmetry and $t \bar{t}$ enhancement data in the fit affects the result only mildly, leaving the conclusions unchanged.

The EFT parameter space that fits the data can also be used to give predictions for future LHC measurements at $8 \mathrm{TeV}$. We focus on two observables: the differential charge asymmetry and the differential $t \bar{t}$ cross section. In figure 3 we show the NP contribution to the former, while figure 4 presents the latter, as described by a modified $\mathcal{S}$ parameter as a function of the cutoff $M_{t \bar{t}}$, along the lines of eq. (4.1). We learn that the heavy NP explanation for the top forward-backward asymmetry predicts an enhancement of the $t \bar{t}$ differential cross section at high $M_{t \bar{t}}$, as was first pointed out in [15]. For example, for $M_{t \bar{t}}>1.5 \mathrm{TeV}$, the minimal enhancement is a factor of 2 above the SM cross section prediction. We also learn that, in the EFT context, the Tevatron $A_{\mathrm{FB}}^{t \bar{t}}$ predicts a positive $A_{\mathrm{C}}$ which grows with energy up to $M_{t \bar{t}} \simeq 800 \mathrm{GeV}$. 


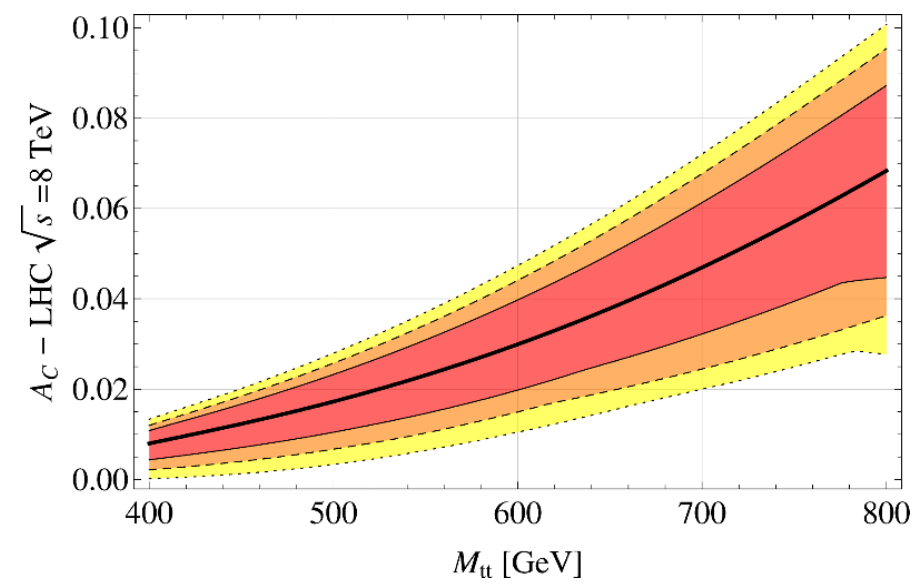

Figure 3. Prediction for the NP contribution (not including the SM) to the differential charge asymmetry as a function of $M_{t \bar{t}}$ for the $8 \mathrm{TeV}$ LHC run. The red, orange and yellow shaded regions correspond to $1 \sigma, 2 \sigma$ and $3 \sigma$ of the global fit to Tevatron data, respectively.

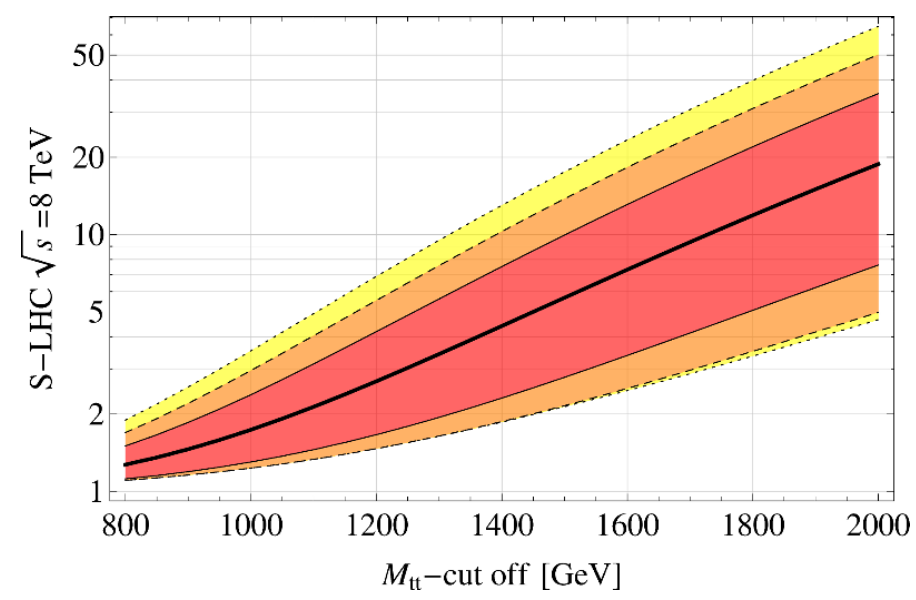

Figure 4. Prediction for the enhancement of the differential $t \bar{t}$ cross section as a function of the cutoff $M_{t \bar{t}}$ for the $8 \mathrm{TeV}$ LHC run, parameterized by the appropriate modification of eq. (4.1). The red, orange and yellow shaded regions correspond to $1 \sigma, 2 \sigma$ and $3 \sigma$ of the global fit to Tevatron data, respectively.

\section{Dijet constraints}

Dijets searches are sensitive to NP dynamics with sizable couplings to light flavors or gluons. Since agreement with the expected SM rate has been observed thus far [30, 31], the available LHC dijet data provides constraints on NP models explaining the top $A_{\mathrm{FB}}^{t \bar{t}}$. In the context of heavy NP, the most naive interpretation of the operator basis defined in section 2 does not generate a dijet signal from a single operator, as the top quark in general escapes the cuts used in the relevant measurement.

If the underlying theory is $\mathrm{SU}(2)_{L}$-invariant, then in a chiral basis couplings between first generation quarks and left-handed $b$ quarks are automatically present. We conservatively assume that the data does not differentiate between the various (non top) quark 


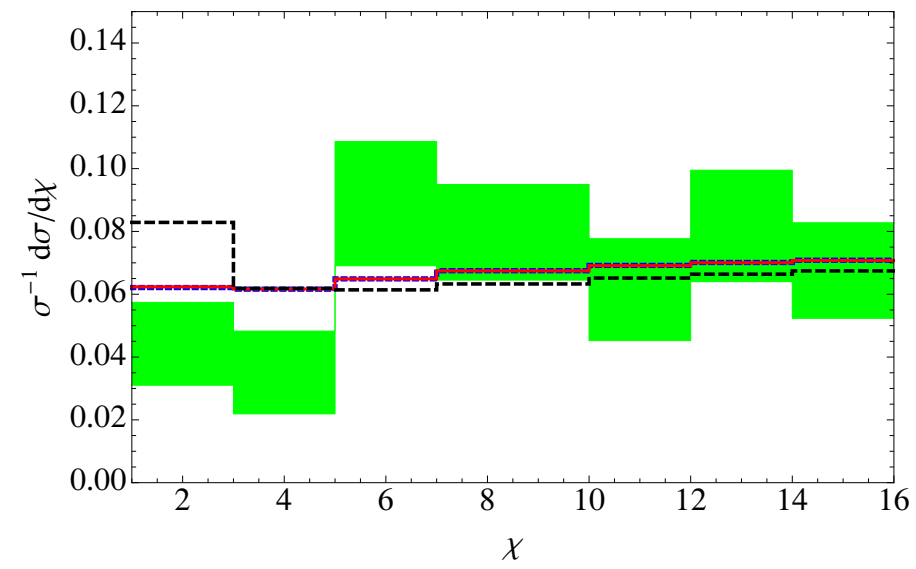

Figure 5. Various contributions at leading order and at the partonic level to the angular distribution of dijet production with $M_{j j}>3 \mathrm{TeV}$ : The SM (dotted blue); heavy NP with $c_{A}^{8}=1.35 \mathrm{TeV}^{-2}$ and all other operators set to zero (solid red); and the limiting operator $+2 \pi /(8 \mathrm{TeV})^{2}\left(\bar{q}_{L} \gamma^{\mu} q_{L}\right)^{2}$ of [30] (dashed black). The measured $1 \sigma$ bands are depicted in shaded green.

flavors, such that $b$ jets are bounded by the current dijet data. Our procedure is as follows. Any point in the fit parameter space can be projected onto the chiral basis, where left handed tops and $b$ 's are interchangeable (we consider both singlet and triplet EW contractions). The angular distribution of the resulting $u \bar{u} \rightarrow b \bar{b}$ contribution to dijet production can then be calculated and compared to the data. In figure 5 we demonstrate this using $c_{A}^{8}=1.35 \mathrm{TeV}^{-2}$ and all other operators turned off, which is representative of the parameters best fitting the Tevatron data. We use a singlet EW contraction, and have checked that a triplet contraction (as well as adding $d \bar{d}$ contributions) does not alter the results. The contribution to dijet production using the CMS method [30] is shown at the partonic level along with the measurement and the SM contribution to the high dijet invariant mass region, $M_{j j}>3 \mathrm{TeV}$. Note that this analysis is only valid for a heavy NP scale above $3 \mathrm{TeV}$, otherwise a model-specific calculation is required. Also shown is the distribution coming from the operator $\left(\bar{q}_{L} \gamma^{\mu} q_{L}\right)^{2}$, where $q_{L}$ denotes the first generation quark left-handed doublet, taken at its limiting value as found in [30]. It is evident that the heavy NP contribution tightly follows that of the SM, and is significantly below the limiting contribution that defines the bound. Hence, this analysis leaves the EFT parameter space unconstrained.

At the loop level, dijets are unavoidably produced by moving to a chiral basis and exchanging a $W$ between left-handed tops, resulting in an effective coupling to down type quarks. The dominant contribution to this dijet signal comes from final state $b$ quarks. As this signal is necessarily smaller than that of the unconstrained tree level process discussed above, we conclude that there are no model-independent dijet constraints on the heavy NP parameter space.

If the operators emerge from an s-channel exchange of a new heavy color octet particle with different axial couplings to up $\left(g_{u u}\right)$ and top quarks $\left(g_{t t}\right)$, then dijets are unavoidably produced [15]. Combining the top $A_{\mathrm{FB}}^{t \bar{t}}$ and dijet data sets a bound on the ratio of the 
couplings:

$$
g_{u u} / g_{t t} \lesssim 1 / 6
$$

In deriving eq. (5.1) we use the latest CMS bound reported for an axial color singlet operator [30], where the operator is rescaled by a factor of $1 / N_{c}$ to account for the different color contraction.

The simplest version of the above NP model is the case of a heavy axigluon. Such a colored octet state with a mass $m_{A}$ and purely axial couplings to quarks induces only the $\mathcal{O}_{A}^{8}$ operator at low energies, and can easily accommodate the preferred data region in figure 1 with $c_{A}^{8} \equiv g_{u u} g_{t t} / m_{A}^{2} \simeq 1 \mathrm{TeV}^{-2}$. This can be achieved with $\mathcal{O}(1)$ perturbative couplings also satisfying the dijet constraint of eq. (5.1) and mass $m_{A}$ of order a few $\mathrm{TeV}$, above the present reach of the LHC. We have also verified that a lighter axigluon with $m_{A} \sim 2 \mathrm{TeV}$, which can be produced on-shell at the LHC, is not excluded by the dijet data (although the ratio of its couplings $g_{u u} / g_{t t}$ must be somewhat smaller than the upper bound in eq. (5.1) in order to account for the Tevatron $A_{\mathrm{FB}}^{t \bar{t}}$ ).

Dijets can also arise by sewing together two operators to generate a $u \bar{u} \rightarrow u \bar{u}$ process through a top loop [32]. However, this loop is quadratically divergent, and so the corresponding constraints are highly model-dependent. For instance, if the $\mathcal{O}_{A}^{8}$ operator arises from the exchange of a massive spin one state, gauge invariance would protect against the quadratic divergence.

Similarly, contributions to atomic parity violation and $Z \rightarrow b \bar{b}$ can emerge through quadratically divergent top and up loops, respectively. In addition to these processes being model-dependent, they can only stem from color-singlet operators, which have a mild impact on the above results.

\section{Implications for $b \bar{b}$ production asymmetry}

We now discuss the effect of the EFT operators considered in this work on the forwardbackward asymmetry in $b \bar{b}$ production. If the underlying theory is $\mathrm{SU}(2)_{L}$-invariant then couplings between first generation quarks and left-handed $b$ quarks necessarily emerge, as mentioned in the previous section.

In figure 6 we show the maximal forward-backward production asymmetry of the $b \bar{b}$ system, $A_{\mathrm{FB}}^{b \bar{b}}$, that can be obtained for $c_{A}^{8}=1.35 \mathrm{TeV}^{-2}$ and all other operators set to zero. This is obtained with an $\mathrm{SU}(2)_{L}$ singlet contraction; the triplet contraction leads to a smaller asymmetry. For other points in the fit region the relative change in the result is at most of $\mathcal{O}(1)$. The asymmetry is plotted as a function of $M_{b \bar{b}}$ according to the binning reported by $\mathrm{CDF}[33]$.

We find that the NP contribution to the asymmetry at invariant mass of up to $200 \mathrm{GeV}$ cannot exceed a permil. In particular, a measurement of $A_{\mathrm{FB}}^{b \bar{b}}$ of order a percent would be inconsistent with this prediction. We conclude that if a large $A_{\mathrm{FB}}^{b \bar{b}}$ is measured, a heavy NP theory which accounts for the top forward-backward asymmetry must include an additional source for the bottom asymmetry beyond the $\mathrm{SU}(2)_{L}$-completion of the operators of eqs. (2.1) and (2.2). 


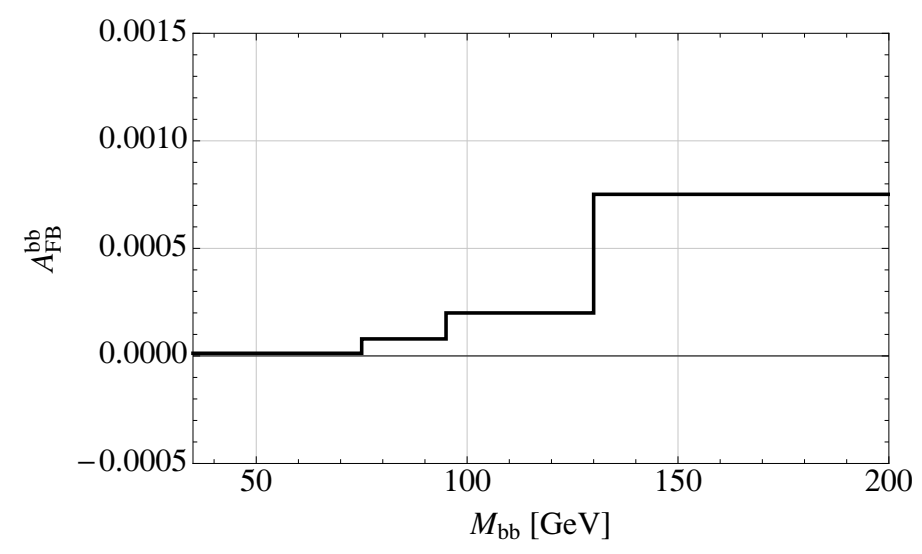

Figure 6. Prediction for the NP contribution to $b \bar{b}$ forward-backward asymmetry for $c_{A}^{8}=$ $1.35 \mathrm{TeV}^{-2}$ and all other operators set to zero.

\section{Conclusions}

We have performed a model independent analysis for the $t \bar{t}$ forward-backward asymmetry and other top-related measurements, assuming heavy new physics. We have shown that this framework is still largely consistent with all the available data. We provide robust predictions for both LHC and Tevatron observables, which will be tested in the near future. Specifically, we predict an enhancement at high invariant masses of both the top pair production cross section and the charge asymmetry at the LHC. Additionally, the $b \bar{b}$ forward-backward asymmetry at the Tevatron cannot exceed $\mathcal{O}\left(10^{-3}\right)$ at invariant mass of up to $200 \mathrm{GeV}$.

The 2012 LHC run should be able to probe the parameter space of the heavy new physics. In particular, since the existing CMS measurement of the differential cross section already places partial constraints on the heavy new physics hypothesis, we expect the $8 \mathrm{TeV}$ data to improve the bounds. This will require the use of dedicated jet reconstruction techniques, since for high invariant masses the top quark is highly boosted, and its decay products typically merge into a single (fat) jet. These new techniques are showing constant improvement (see e.g. [29, 34-36]), and will soon be able to provide a partonic level measurement of the $M_{t \bar{t}}$ distribution in the boosted regime, where $\mathrm{TeV}$ scale new physics might be at play.

\section{Acknowledgments}

We thank Christophe Grojean, Alex Kagan, Gilad Perez and Ofer Vitells for useful discussions, and Yossi Nir for comments on the manuscript.

Open Access. This article is distributed under the terms of the Creative Commons Attribution License which permits any use, distribution and reproduction in any medium, provided the original author(s) and source are credited. 


\section{References}

[1] CDF collaboration, T. Aaltonen et al., Evidence for a mass dependent forward-backward asymmetry in top quark pair production, Phys. Rev. D 83 (2011) 112003 [arXiv:1101.0034] [INSPIRE].

[2] CDF collaboration, Measurement of the forward backward asymmetry in top pair production in the dilepton decay channel using $5.1 \mathrm{fb}^{-1}$, CDF note 10436, Fermilab, Batavia U.S.A. March 102011.

[3] D0 collaboration, V.M. Abazov et al., Forward-backward asymmetry in top quark-antiquark production, Phys. Rev. D 84 (2011) 112005 [arXiv:1107.4995] [INSPIRE].

[4] CDF collaboration, Study of the top quark production asymmetry and its mass and rapidity dependence in the full run II Tevatron dataset, CDF note 10807, Fermilab, Batavia U.S.A. March 72012.

[5] J.H. Kuhn and G. Rodrigo, Charge asymmetries of top quarks at hadron colliders revisited, JHEP 01 (2012) 063 [arXiv:1109.6830] [INSPIRE].

[6] S.J. Brodsky and X.-G. Wu, Application of the principle of maximum conformality to the top-quark forward-backward asymmetry at the Tevatron, Phys. Rev. D 85 (2012) 114040 [arXiv: 1205.1232] [INSPIRE].

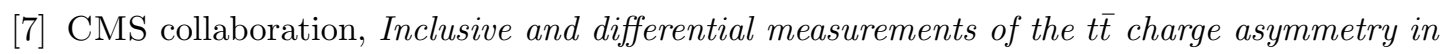
proton-proton collisions at 7 TeV, Phys. Lett. B 717 (2012) 129 [arXiv:1207.0065] [INSPIRE].

[8] ATLAS collaboration, Measurement of the charge asymmetry in top quark pair production in pp collisions at $\sqrt{s}=7$ TeV using the ATLAS detector, Eur. Phys. J. C 72 (2012) 2039 [arXiv: 1203.4211] [INSPIRE].

[9] ATLAS collaboration, Measurement of the charge asymmetry in dileptonic decay of top quark pairs in pp collisions at $\sqrt{s}=7$ TeV using the ATLAS detector, ATLAS-CONF-2012-057, CERN, Geneva Switzerland June 22012.

[10] CMS collaboration, Measurement of top quark pair differential cross sections at $\sqrt{s}=7 \mathrm{TeV}$, CMS-PAS-TOP-11-013, CERN, Geneva Switzerland February 82012.

[11] D.-W. Jung, P. Ko, J.S. Lee and S.-H. Nam, Model independent analysis of the forward-backward asymmetry of top quark production at the Tevatron, Phys. Lett. B 691 (2010) 238 [arXiv:0912.1105] [INSPIRE].

[12] C. Degrande, J.-M. Gerard, C. Grojean, F. Maltoni and G. Servant, Non-resonant new physics in top pair production at hadron colliders, JHEP 03 (2011) 125 [arXiv:1010.6304] [INSPIRE].

[13] D.-W. Jung, P. Ko and J.S. Lee, Longitudinal top polarization as a probe of a possible origin of forward-backward asymmetry of the top quark at the Tevatron, Phys. Lett. B 701 (2011) 248 [arXiv: 1011.5976] [INSPIRE].

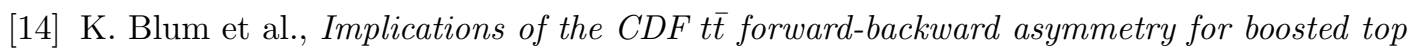
physics, Phys. Lett. B 702 (2011) 364 [arXiv:1102.3133] [INSPIRE].

[15] C. Delaunay, O. Gedalia, Y. Hochberg, G. Perez and Y. Soreq, Implications of the CDF $t \bar{t}$ forward-backward asymmetry for hard top physics, JHEP 08 (2011) 031 [arXiv:1103.2297] [INSPIRE]. 
[16] J. Aguilar-Saavedra and M. Pérez-Victoria, Probing the Tevatron $t \bar{t}$ asymmetry at LHC, JHEP 05 (2011) 034 [arXiv: 1103.2765] [INSPIRE].

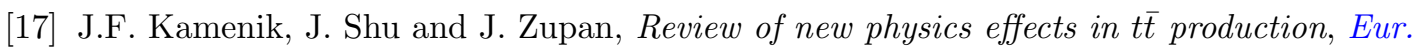
Phys. J. C 72 (2012) 2102 [arXiv:1107.5257] [INSPIRE].

[18] R. Barbieri and A. Strumia, The 'LEP paradox', hep-ph/0007265 [InSPIRE].

[19] R. Barbieri, A. Pomarol, R. Rattazzi and A. Strumia, Electroweak symmetry breaking after LEP-1 and LEP-2, Nucl. Phys. B 703 (2004) 127 [hep-ph/0405040] [InSPIRE].

[20] ALEPH, DELPHI, L3, OPAL, SLD, LEP Electroweak Working Group, SLD Electroweak Group, SLD Heavy Flavour Group collaborations, S. Schael et al., Precision electroweak measurements on the $Z$ resonance, Phys. Rept. 427 (2006) 257 [hep-ex/0509008] [INSPIRE].

[21] J. Drobnak, J.F. Kamenik and J. Zupan, Flipping $t \bar{t}$ asymmetries at the Tevatron and the LHC, Phys. Rev. D 86 (2012) 054022 [arXiv: 1205.4721] [InSPIRE].

[22] D0 collaboration, V.M. Abazov et al., Measurement of the t $\bar{t}$ production cross section using dilepton events in pp collisions, Phys. Lett. B 704 (2011) 403 [arXiv:1105. 5384] [INSPIRE].

[23] P. Baernreuther, M. Czakon and A. Mitov, Percent level precision physics at the Tevatron: first genuine NNLO QCD corrections to $q \bar{q} \rightarrow t \bar{t}+X$, Phys. Rev. Lett. 109 (2012) 132001 [arXiv: 1204.5201] [INSPIRE].

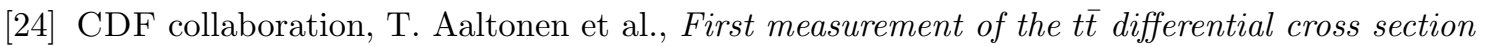
$d \sigma / d M(t \bar{t})$ in p p collisions at $\sqrt{s}=1.96$ TeV, Phys. Rev. Lett. 102 (2009) 222003 [arXiv:0903.2850] [INSPIRE].

[25] V. Ahrens, A. Ferroglia, M. Neubert, B.D. Pecjak and L.L. Yang, Renormalization-group improved predictions for top-quark pair production at hadron colliders, JHEP 09 (2010) 097 [arXiv: 1003.5827] [INSPIRE].

[26] A. Martin, W. Stirling, R. Thorne and G. Watt, Parton distributions for the LHC, Eur. Phys. J. C 63 (2009) 189 [arXiv:0901.0002] [InSPIRE].

[27] B. Grinstein, A.L. Kagan, M. Trott and J. Zupan, Forward-backward asymmetry in $t \bar{t}$ production from flavour symmetries, Phys. Rev. Lett. 107 (2011) 012002 [arXiv:1102.3374] [INSPIRE].

[28] F. del Aguila, J. de Blas and M. Pérez-Victoria, Electroweak limits on general new vector bosons, JHEP 09 (2010) 033 [arXiv: 1005.3998] [INSPIRE].

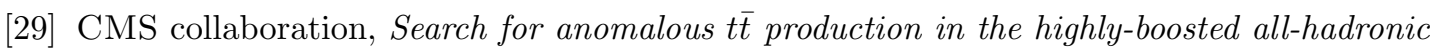
final state, JHEP 09 (2012) 029 [arXiv:1204.2488] [INSPIRE].

[30] CMS collaboration, Search for quark compositeness in dijet angular distributions from pp collisions at $\sqrt{s}=7 \mathrm{TeV}$, JHEP 05 (2012) 055 [arXiv: 1202.5535] [INSPIRE].

[31] ATLAS collaboration, Search for new physics in dijet mass and angular distributions using $4.8 \mathrm{fb}^{-1}$ of pp collisions at $\sqrt{\mathrm{s}}=7 \mathrm{TeV}$ collected by the ATLAS detector, ATLAS-CONF-2012-038, CERN, Geneva Switzerland March 142012.

[32] O. Domenech, A. Pomarol and J. Serra, Probing the SM with dijets at the LHC, Phys. Rev. D 85 (2012) 074030 [arXiv:1201.6510] [INSPIRE].

[33] P. Bartos, $b \bar{b}$ asymmetry at CDF, CDF talk at the CERN AFB workshop, April 2012. 
[34] CDF collaboration, T. Aaltonen et al., Study of substructure of high transverse momentum jets produced in proton-antiproton collisions at $\sqrt{s}=1.96 \mathrm{TeV}$, Phys. Rev. D 85 (2012) 091101 [arXiv: 1106 .5952] [INSPIRE].

[35] ATLAS collaboration, Jet mass and substructure of inclusive jets in $\sqrt{s}=7 \mathrm{TeV} p p$ collisions with the ATLAS experiment, JHEP 05 (2012) 128 [arXiv:1203.4606] [INSPIRE].

[36] ATLAS collaboration, Measurement of event shapes at large momentum transfer with the ATLAS detector in pp collisions at $\sqrt{s}=7 \mathrm{TeV}$, arXiv:1206.2135 [INSPIRE]. 